461 INVESTIGATING EPIGENOMIC INVOLVEMENT IN ACQUIRED DRUG-RESISTANCE IN HIGH-GRADE SEROUS OVARIAN CANCER

${ }^{1,2,3} \mathrm{R}$ Silva*, ${ }^{2} \mathrm{M}$ Metoudi, ${ }^{2,4} \mathrm{~K}$ Glennon, ${ }^{1,3} \mathrm{~A}$ Perry, ${ }^{2,4} \mathrm{D}$ Brennan. ${ }^{1}$ University College Dublin, Cancer Biology and Therapeutics Laboratory, UCD Conway Institute of Biomolecular and Biomedical Research, Ireland; ' ${ }^{2}$ niversity College Dublin, Systems Biology Ireland, UCD School of Medicine, Ireland; ${ }^{3}$ University College Dublin, School of Biology and Environmental Science, Ireland; ${ }^{4}$ Mater Misericordiae University Hospital, Department of Gynaecological Oncology, Ireland

\subsection{6/ijgc-2021-ESG0.594}

Introduction/Background* Due to advanced presentation at diagnosis and subsequent development of therapeutic resistance, ovarian cancer (OC) remains the most lethal gynaecological malignancy worldwide. High-grade serous ovarian cancer (HGSOC) is the predominant OC subtype observed in the clinical setting, and approximately $80 \%$ of women eventually develop therapeutic resistance to the standard platinum therapy regimen. The aim of this study is to understand how the epigenomic events unfold during acquired-drug resistance in HGSOC patients.

Methodology A HGSOC publicly-available dataset was used to explore alterations between primary platinum-sensitive samples $(\mathrm{n}=32)$ and recurrent acquired resistant samples $(n=28)$. Results derived from methylation (HM450K array) and gene expression (RNAseq) analysis were further investigated in a paired-sample subset of the cohort $(n=12)$. Validation, using high resolution melting and Sanger sequencing, was carried out using two pairs of matched platinum-sensitive and resistant cell lines (A2780 and UWB1.289), along with samples from pre- and post-platinum treated HGSOC patients $(\mathrm{n}=3)$.

Result(s)* Comparison of methylation and gene expression analysis identified several genes, known to be involved in diverse immune and chemoresistance-related pathways, that significantly differentiated between paired platinum-sensitive and acquired resistant samples: PDCD1, THY1, NKAPL, C1QTNF4, DIO3, EOMES, APOBEC3A and S100A8. A detailed evaluation of the paired cohort suggested that epigenomic alterations in these genes were associated with time to relapse. Further investigation into the methylation status of these markers in paired cell lines and HGSOC patient samples revealed change in methylation dynamics (i.e. increased/ decreased methylation) across the majority of markers, in response to treatment with platinum compounds. Of the studied markers, PDCD1, NKAPL and APOBEC3, known to be prognostic markers in OC, displayed the most consistent changes across all samples. Additionally, we observed marked methylation differences between primary and metastatic samples from the same patient, highlighting the heterogeneity of the disease and the potential use of liquid biopsies to explore whole-tumour dynamics.

Conclusion* Initial investigations have identified a panel of epigenetic alterations potentially involved in HGSOC drug resistance. Dynamic methylation changes and response to platinum treatment will be further evaluated in a longitudinal plasma cell-free DNA cohort of HGSOC patients.

\section{CANCER ORGANOID MODELS ARE VERY HETEROGENEOUS AND LACK ROBUSTNESS FOR CLINICAL POPULATIONS}

${ }^{1,2} \mathrm{Z}$ Jan*, ${ }^{2} \mathrm{R}$ Heremans, ${ }^{2} \mathrm{~N}$ Maenhoudt, ${ }^{2} \mathrm{H}$ Vankelecom, ${ }^{2} \mathrm{D}$ Timmerman. ${ }^{1}$ Cancer centre Carinthia, Klinikum Klagenfurt, Klagenfurt, Austria; ${ }^{2} \mathrm{KU}$ Leuven (University of Leuven), Development and Regeneration, Leuven, Belgium

\subsection{6/ijgc-2021-ESG0.595}

Introduction/Background* Cancer organoids are an emerging in vitro tool to study tumorigenesis.

However, patient-specific applications, s. a. chemotherapy response prediction, remain understudied.

Organoid protocols vary considerably between different publications, impeding clinical applications.

Our aim was to examine the experimental design characteristics in current human gynaecologic cancer (GC) organoid studies.

Methodology We conducted a focused review and included publications on GC organoids in the analysis that recruited $\geq$ 3 patients.

Result(s)* In 7 studies, 178 patients with ovarian cancer had $\geq 1$ biopsy, establishing 163 organoid cultures. Of all patients, 87 had high-grade serous histology. 22\% of all biopsies were taken from chemotherapy-naïve patients. Organoid culture success rate ranged between 30-100\%.

40 organoid cultures could be established from patients in 4 studies on endometrial cancers. Organoid culture success rate was inconsistently reported and ranged between $42-83 \%$. Most cancers $(45 \%, 18 / 40)$ were of low-grade endometrioid histology, however molecular classification was not provided.

In a single study, 22 patients with squamous cervical cancer and 4 with adenocarcinoma gave rise to 11 and 1 successful organoid culture, respectively.

To date, there are no data on human vulvar, vaginal, or malignant trophoblast organoids.

In a minority (2/12) of the studies, percentage of tumour cells in the biopsy or cell vitality was reported.

The growth factor supplementation was rarely tested for $\geq 3$ formulations of GC organoid culture media, making a comparison between protocols difficult. In addition, different media weren't allocated to biopsies randomly. Neither the experimental conditions nor the outcomes were blinded to researchers in any of the studies.

Conclusion* Most of the current human GC organoid studies are observational studies and do not employ statistical preplanning for clinical subpopulations. There appears a general underreporting for failed organoid cultures. Due to heterogeneous experimental designs, robust organoid media are not available yet. 